\title{
L'ÉGLISE, LES COPTES ET LA RÉVOLUTION DU 25 JANVIER : MUTATIONS COMMUNAUTARISTES OU SORTIE DE LA LOGIQUE IDENTITAIRE ?
}

\author{
Laure Guirguis
}

Centre d'études et de recherches sur le Proche-Orient | « Les Cahiers de l'Orient »

2012/4 N 108 | pages 115 à 132

ISSN 0767-6468

Article disponible en ligne à l'adresse :

https://www.cairn.inforevue-les-cahiers-de-l-orient-2012-4-page-115.htm

Distribution électronique Cairn.info pour Centre d'études et de recherches sur le Proche-Orient.

(C) Centre d'études et de recherches sur le Proche-Orient. Tous droits réservés pour tous pays.

La reproduction ou représentation de cet article, notamment par photocopie, n'est autorisée que dans les limites des conditions générales d'utilisation du site ou, le cas échéant, des conditions générales de la licence souscrite par votre établissement. Toute autre reproduction ou représentation, en tout ou partie, sous quelque forme et de quelque manière que ce soit, est interdite sauf accord préalable et écrit de l'éditeur, en dehors des cas prévus par la législation en vigueur en France. Il est précisé que son stockage dans une base de données est également interdit. 


\title{
L'Église, les coptes et la révolution du 25 janvier :
}

\author{
mutations communautaristes \\ ou sortie de la logique identitaire?
}

Par Laure Guirguis*

Tatalysant les émotions contestataires, la figure du
jeune Khâlid Saî̀d incarna initialement l'opposi-
tion au régime sécuritaire qualifié d'ennemi national. L'expérience révolutionnaire unifia momentanément les demandes sectorielles. Le peuple semblait assister à sa propre naissance et se contemplait en slogans, en vidéos et en chants. Pourtant, dès le mois de février 2011, lorsque se produisirent les premières échauffourées entre coptes, salafistes et militaires, l'événement révolutionnaire réactiva des dispositifs structurels, et en particulier la communautarisation de la société et de la vie politique égyptiennes. Structurels car ces dispositifs, héritages d'une longue histoire, reproduits tout en étant profondément modifiés au cours des siècles, gouvernent actuellement les relations entre les individus, et entre les individus et l'État, selon des modalités éminemment contemporaines. Initiées par les règles et les pratiques des époques pré-républicaines, les discriminations exercées à l'encontre des chré-

\footnotetext{
* Laure Guirguis, titulaire d'une bourse postdoctorale Banting en science politique à l'Université de Montréal, a travaillé sur le fait minoritaire copte en Égypte. Elle est l'auteure de [Coptes d'Égypte et reconfigurations politiques, 2005-2012]. Ses recherches actuelles portent sur les processus de minorisation et la figuration du martyr en Égypte, au Liban et en Israël/Palestine.
} 
tiens ne se réferent à la religion, perçue comme marqueur identitaire, qu'à partir du choc de la confrontation avec les grandes puissances au XIX siècle et de l'émergence des nationalismes qui s'ensuivit. L'ère nassérienne, apogée des nationalismes arabe et égyptien, cristallise ensuite dans l'État-nation sécuritaire les logiques identitaires présidant au maintien, à la reconduction et à la transformation des processus de minorisation. Au lendemain de la défaite de 1967, l'avènement de Sadate, représentant de l'aile droite religieuse et conservatrice des Officiers libres, marque la victoire de la variante religieuse de l'idéologie identitaire et le début du long déclin de l'idéal national.

La révolution du 25 janvier 2011 exacerba l'antagonisme, qui tiraille les coptes depuis plus d'un siècle, entre la tentation du repli derrière les murs de l'Église devenue monde sous haute protection patriarcale et l'engagement dans l'action politique sur la scène nationale. Mais elle révélait également l'inanité de la logique identitaire commune aux deux termes de l'alternative, nationalisme et communautarisme. Au lendemain de cet épisode critique, assiste-t-on aux prémices de la sortie de la logique identitaire ou à sa reproduction selon des modalités inédites, qui incluent la pluralité et la dimension transnationale?

Révélatrice des tendances lourdes, structurelles - la communautarisation, la centralité de la question identitaire par le prisme des normativités islamiques, etc. - la révolution précipita en effet la formation ou la transformation de courants et de représentations diffus. Avivant les dissensions générationnelles, idéologiques et tactiques qui taraudaient toutes les organisations et institutions, elle dévoila la pluralité et la multiplication des centres d'autorité - politique et religieuse, dans les mondes copte et musulman - jusqualors partiellement masquées par l'absence de structures politiques représentatives. À l'instar d'al-Azhar, l'Église copte se voit sommée de redéfinir ses relations avec l'État et les dirigeants, de repenser l'organisation de l'institution cléricale, de se positionner par rapport à la production d'une multitude de discours religieux concurrents - parfois au sein même de l'orthodoxie - 
1. Anciennement membre de la Jamâ'a islâmiyya au Caire, il rejoignit les Frères musulmans dans les années 1970 et fut membre du Conseil consultatif (Majlis al-chûra) ainsi que du bureau politique de la Confrérie. Il était surtout chargé des questions médiatiques et ne disposait pas d'un pouvoir décisionnel.

Député de 1987 à 1990, il fait partie de la génération dite intermédiaire des Frères, qui a contribué à leur ascension dans les syndicats professionnels à la fin des années 1980. Il est actuellement membre de la direction du Parti Liberté et Justice.

2. Fondateur de la confrérie des Frères musulmans (1906-1949). et de faire face aux rivalités intercléricales ou aux dissensions infracommunautaires.

Les dynamiques transnationales et les recompositions des discours et pratiques au sein de l'islam joueront par conséquent un rôle crucial dans l'évolution de la situation des chrétiens en Égypte. Mais les facteurs nationaux s'avéreront plus déterminants, au moins sur le court terme. Or, pour l'heure, deux inconnues majeures subsistent, dès lors que les données actuelles permettent difficilement d'esquisser les contours de l'appareil d'État en cours de restructuration, non plus que ceux d'une Église en crise que le successeur de Chenouda III devra réformer.

\section{L'Église et la révolution}

Le patriarche Chenouda III (1971-2012) soutint le régime et le PND pendant les journées insurrectionnelles qui décidèrent les officiers à pousser Hosni Moubarak vers la sortie, le 11 février 2011. Deux jours plus tard, non sans avoir attendu, d'après plusieurs observateurs, d'être certain que le départ du râs était définitif, le patriarche salua la "courageuse armée d'Égypte » et la « jeunesse honnête du 25 janvier qui [avait] mené une révolution puissante» et "offert son sang ", puis se prononça en faveur d'un "État civil (madanî) démocratique " et "d'élections libres". S'interrogeant sur les critères qui régiraient la création des partis, Chenouda III exprima d'emblée, mais à mots couverts, la crainte copte d'une ascension politique des Frères musulmans. Hanî 'Azîz, homme d'affaires copte proche du patriarche et membre du PND, formula à son tour l'inquiétude des coptes au sujet des intentions des Frères, en signalant que 'Isâm al-'Iryân ${ }^{1}$ avait, sur les chaînes satellitaires, pris la parole devant un grand portrait de Hasan al-Bannầ ${ }^{2}$.

En l'absence de parti régnant dans la compétition pour siéger sous la coupole du Parlement, l'Église donna des consignes de vote en faveur de la Kutla, la coalition incluant le Parti des Égyptiens libres (Hizb al-misriyyûn al-ahrâr) éta- 
bli après la révolution par l'homme d'affaires copte Nagub Sawîrîs. Confronté à la victoire électorale des partis islamistes, Chenouda III prêta allégeance au nouveau dirigeant élu: il convia les Frères musulmans et les partis salafistes à la célébration de la fête de Noël et manifesta son respect à l'égard du pouvoir militaire dont la légitimité forgée dans le moment révolutionnaire s'était érodée au fil des mois de répression. En d'autres termes, les reconfigurations de la scène politique égyptienne, l'émergence de nouvelles figures et l'équilibre de forces partiellement inédit issu de ces transformations n'ayant, pour l'heure, abouti ni à une refonte de l'appareil d'État ni à une redéfinition des rapports entre les institutions étatiques et les gouvernants, les motifs structurels qui contraignent l'Église à se ranger aux côtés du régime, quel qu'il soit, demeurent inchangés.

De fait, l'Église, en tant qu'institution d'État, et indépendamment des orientations politiques de l'élite cléricale, est tributaire des services de la sûreté de l'État (Amn al-dawla) pour assurer la protection des fidèles, des terres et des lieux de culte - quelque insatisfaisante que soit la manière dont les institutions étatiques s'acquittent de cette tâche - dans un pays où les discours de haine et la défiance tissent le quotidien des relations entre les adeptes des deux religions, tandis que les épisodes de violence se produisent à une cadence accélérée depuis quatre décennies. Elle est soumise directement au bon vouloir du chef de l'État, des agents des institutions policières et des administrations provinciales pour la délivrance des permis de construire et de restaurer les lieux de culte. Enfin, en dépit de l'autonomie législative limitée et contrôlée dont elle jouit dans le domaine du statut personnel des chrétiens, ce sont les tribunaux étatiques, et non plus confessionnels, qui tranchent dans les cas de divorce et de (re)conversion au christianisme, mettant en jeu la sphère d'autorité impartie à l'Église. Or, depuis le coup d'État des Officiers libres au mois de juillet 1952, l'État égyptien se caractérise par l'absence d'indépendance des institutions et des agents étatiques - judiciaire inclus. Corrélativement, les régimes présidentiels successifs instaurèrent le règne de l'informel autorisant l'arbi- 
traire. Les individus et les groupes ne doivent par conséquent leur sauvegarde qu’à leurs accointances avec le régime, soit, jusqu'au mois de février 2011, avec le parti au pouvoir.

La connivence entre l'Église et le régime ne reposait pas simplement sur le quasi statut de représentant politique des coptes conféré au patriarche dès l'ère nassérienne, non plus que sur le seul soutien accordé par l'Église à la politique gouvernementale. Elle s'était encore instituée au travers de multiples réseaux d'alliances et de solidarité entre le haut clergé et les élites économico-politiques (proches) du PND. Suivant une évolution similaire à celle constatée sur la scène politique nationale, les hommes d'affaires grossirent les rangs de l'entourage du patriarche. Certes, sous la présidence de Sadate, les rapports entre les leaders communautaire et national furent rythmés par des épisodes de confrontation qui se soldèrent par la destitution arbitraire du chef de l'Église en 1981, principalement motivée par le refus de Chenouda de soutenir le plan de paix présidentiel avec l'État d'Israël. Au retour de son exil forcé dans le Wadi Natroun - depuis lequel il avait continué à administrer officieusement les affaires de l'Église - le patriarche avait adopté une attitude plus conciliante à l'égard d'un régime décidé à réprimer les groupes islamistes radicaux. Pour autant, ni les épisodes de confrontation renouvelés au cours de la dernière décennie, ni ceux qui marquèrent le règne de Sadate, ne remirent en question l'alliance fondamentale contractée entre les gouvernants et le patriarche, quelque biaisés que soient les termes de ce pacte tacite. Plus loin, l'opposition patriarcale à une décision gouvernementale ou étatique fut souvent déterminée par des dissensions infracommunautaires et par le souci de remobiliser la communauté contre un présumé ennemi commun.

\section{Militances coptes et révolution}

La crainte d'une ascension politique des courants islamistes, partagée par la plupart des coptes, se conjugua cependant à des attitudes variées vis-à-vis du soulèvement 
qui embrasa la rue égyptienne à l'aube de l'année 2011. En dépit de cette appréhension, beaucoup de chrétiens fêtèrent le départ de Hosni Moubarak, même s'ils s'étaient auparavant tenus à l'écart du mouvement insurrectionnel. Globalement et schématiquement, les coptes, d'abord inquiets, avaient, comme leurs concitoyens, vu l'horizon se dégager et l'avenir redevenir possible. Pendant les journées révolutionnaires, la majorité des coptes est cependant restée dans l'expectative. Certains manifestèrent leur méfiance à l'égard d'un mouvement qu'ils estimaient noyauté par les Frères et les salafistes, d'aucuns observant la multiplication des barbes sur la place Tahrîr et refusant par conséquent de se joindre à la mobilisation. Néanmoins, le nombre de coptes engagés dans l'action révolutionnaire, notamment les jeunes, loin d'être négligeable, incita l'Église à nuancer sa position et à déclarer par voix non officielle, une semaine après le début de l'insurrection, qu' elle ne s'opposait pas à ce que les fidèles participent à des manifestations pour revendiquer le respect de leurs droits et libertés de citoyens "dans de saines conditions". Au contraire de Chenouda III et de l'Anbẩ Bichuy, l'Anbâ Mûsâ (qui présidait alors l'Évêché de la jeunesse et des affaires sociales) prit publiquement position contre le régime et en faveur du mouvement révolutionnaire avant la démission de Hosni Moubarak.

En revanche, au début du mois de février 2011, sur les sites Internet des associations coptes en diaspora, le temps semblait suspendu. Depuis l'attentat commis à Alexandrie un mois auparavant, rien n'était arrivé. Malgré la véhémence des critiques qu'ils adressaient au régime et les pressions qu'ils tentaient d'exercer sur lui, les militants en diaspora appréhendaient, pour la plupart d'entre eux, la chute d'un gouvernement qui constituait à leurs yeux le dernier rempart contre l'islamisation achevée de la société égyptienne. Le 4 février, toutefois, Coptic Solidarity ${ }^{4}$ publia un communiqué exigeant le départ de Hosni Moubarak, la dissolution de l'Assemblée du peuple (Majlis al-cha'b) à la suite d'un référendum et l'instauration d'un gouvernement de transition dans le respect de la légalité constitutionnelle. En revanche, dès les premiers
3. Terme copte issu de l'araméen abba (père), qui a aussi donné notre abbé : père spirituel d'une communauté religieuse $(\mathrm{NdR})$.

4. Plateforme établie au mois de juin 2010, présidée par Adel Guindy, et visant à fédérer les efforts des diverses associations coptes en diaspora pour la défense des droits des coptes et de la citoyenneté. 
jours, les Églises protestantes (dont la célèbre église de Qasr al-Dubbâra, surnommée "église de la révolution ") prirent part au mouvement révolutionnaire, de même que plusieurs prêtres coptes orthodoxes.

La plupart des chrétiens qui descendirent dans la rue le 25 janvier ou les jours suivants, à l'instar des autres Égyptiens, n'étaient pas affiliés à un parti "d'opposition ", à une association de plaidoyer ou à quelque autre groupe protestataire. Les actions de contestation sectorielles et multiformes qui scandèrent les premières années du XXI siècle avaient réveillé le sens critique de ceux-là mêmes qui n'entendaient pas s'engager en politique en situation autoritaire - tels les badauds qui, en 2005, s'alanguissaient sur les balcons ou s'arrêtaient dans la rue pour observer, une lueur dans le regard, les activistes du mouvement Kifâya désigner le gigantesque portrait d'un Moubarak quadragénaire en hurlant "al-harâmî ahu " ("c'est lui le voleur»). Bien que les coptes se soient, depuis la fin des années 1940, progressivement désintéressés d’une vie politique nationale dont ils se sentaient exclus, la vitalité nouvelle de la contestation sur la scène nationale avait gagné les rangs chrétiens et avait été stimulée par la militance copte en diaspora. Loin d'être les sujets passifs des mutations en cours, les coptes ont contribué depuis plusieurs décennies à la reproduction des processus de communautarisation aussi bien qu'aux actions contestataires visant à briser les frontières communautaires et à promouvoir l'établissement d'un État séculier, ou du moins d'un État de droit. En Égypte, des coptes militants sur la scène communautaire, tels les laïcs, ont inscrit leurs revendications dans le cadre national; d'autres ont grossi les rangs de mouvements actifs à l'échelle nationale.

En faible proportion dans les milieux ouvriers, les coptes ont peu participé aux vagues de grèves qui se succédèrent dans les usines égyptiennes. Mais ceux qui prirent part à ces actions durent souvent enfreindre les directives de l'Église. Un chrétien coordonna en 2007 la longue grève des collecteurs de l'impôt foncier, un autre celle chemins de fer. Le spectacle quotidien, ou presque, des mobilisations ouvrières depuis 2006, leurs résultats parfois positifs et la politisation 
des revendications eurent un impact décisif sur la lente maturation du mouvement révolutionnaire. Ils familiarisèrent les Égyptiens avec l'idée que la contestation était non seulement possible, mais parfois, dans une certaine mesure, couronnée de succès. Ces actions constituèrent un " exercice pratique de masse, de la démocratie en action $»^{5}$, la répétition d'un événement encore imprévu.

Mona Mina, figure de proue de Médecins sans droits, une association fondée pour défendre les droits des médecins et pallier ainsi l'inaction du syndicat officiel, et Georges Ishaq, le plus connu des leaders chrétiens de l'opposition, co-fondateur du mouvement Kifâya, se trouvaient place Tahrîr dès le 25 janvier 2011. Présents dans diverses formations situées sur la gauche de l'échiquier politique, les coptes contribuèrent également à l'essor récent des associations de plaidoyer et des ONG égyptiennes. Les membres du groupe MARED ${ }^{6}$, par exemple, figurèrent parmi les révolutionnaires de la première heure. Fondé par Mounir Megahed après les attentats commis à Alexandrie au mois d'avril 2006, ce groupe comprend des chrétiens, des musulmans et des bahaïs. En dépit des obstacles que les agents de la sûreté de l'État et les Frères musulmans opposèrent, parfois conjointement, à leur activité, ils engagèrent deux types d'actions, les unes - conférences et publications - visant à sensibiliser le public à l'existence de discriminations et à leur inanité, les autres - participation à des sit-in, pétitions, publication de communiqués - ayant pour objectif la satisfaction d'une revendication précise formulée en réaction à un événement ponctuel. Travaillant en étroite collaboration avec des associations de plaidoyer et des avocats, ils concentrèrent en particulier leurs efforts sur les affaires de (re)conversion au christianisme et sur le cas des bahầis qui furent portés, massivement, devant les juges égyptiens à partir de l'année 2005.

Cette année-là, la libération de la parole subséquente à l'annonce présidentielle d'amender l'article 76 de la Constitution égyptienne, afin d'organiser des élections à plusieurs candidats, encouragea groupes et individus à dénoncer le caractère autoritaire de la politique patriarcale sur les
5. Cf. infra, l'article de Joel Beinin.

6. Misriyyûn Against Religious Discrimination, (Misriyyûn didd al-tamîyz al-dînî),

[Égyptiens contre les discriminations religieuses]. 
7. Alain

Roussillon,

«Visibilité

nouvelle de la

"question copte" :

entre refus de

la sédition et

revendication

citoyenne ", in

Florian Kosthall

(dir.), L'Égypte

dans l'année 2005 ,

Le Caire, CEDEJ, 2006, p. 137-175.

8. Kamâl Zâkhir, Al-ilmâniyûn wa al-kanîssa. Sirâât wa tahâlufât [Les laïcs et l'Église. Antagonismes et alliances], Le

Caire, s.e., 2009.

9. Revue $R \hat{u} z$ al-Yûsif, $\mathrm{n}^{\circ} 4055$, 25-26 mars 2006.

10. Hânî Labîb,

Al-kanîsa

al-misriyya.

Tawâzunât

al-dîn wa

al-dawla [L'Église

égyptienne.

L'équilibre entre

la religion et

l'État], Le Caire,

Dâr Nahda Misr,

2012. scènes communautaire et nationale. Lors de ce scrutin dont l'organisation inattendue avait éveillé les espoirs, l'injonction patriarcale (formulée peu après une visite de Zakaryâ 'Izmî à Chenouda) à voter pour le candidat sortant souleva de nombreuses critiques, parmi les musulmans comme parmi les coptes ${ }^{7}$. D'abord silencieuses, ou s'exprimant dans le refus parfois informulé de suivre les consignes de vote intimées par le patriarche, ces voix critiques haussèrent le ton. Devenues audibles, elles articulaient fréquemment la dénonciation de la politique du patriarche Chenouda III et du soutien inconditionnel qu'il accordait au régime égyptien à la contestation de l'ordre sécuritaire entretenu par le parti au pouvoir. Elles connurent leur apogée dans le mouvement révolutionnaire. Puis, au mois de janvier 2012, des fidèles clamèrent: " à bas le régime militaire ", en interrompant le patriarche qui adressait ses vœux à l'armée égyptienne dans la prédication délivrée à l'occasion de la fête de Noël.

Le groupe dit " des laïcs » était passé à l'offensive publique dès 2006. Actifs depuis la fin des années 1980, ils inscrivirent résolument leur projet de réforme de l'Église dans le cadre médiatique et politique national. Lors de leur première conférence en 2006, ils exposèrent leurs propositions de réforme de l'institution cléricale, qui impliquaient la revalorisation du rôle des laïcs dans l'administration des affaires de l'Église, tout en les articulant à la promotion de la citoyenneté et de la sécularité. Kamâl Zâkhir, chef de file de cette mouvance, publia les textes des interventions prononcées lors de cette conférence ${ }^{8}$. Divisé encore, ce "groupe de pression [est] sans influence dans le milieu ecclésiastique " ", écrivait Hânî Labîb, membre éphémère de ce courant et auteur d'une histoire contemporaine de l'Église copte ${ }^{10}$.

La jeunesse occupe le devant de la scène contestataire copte depuis le début des années 2000. Et la révolution du 25 janvier, emmenée par le refus des modes d'organisation autoritaire caractéristiques des organismes étatiques et des formations politiques égyptiennes, accéléra les processus de rupture générationnelle qui travaillaient l'Église copte, à l'instar des autres institutions religieuses. Dès 2004, les jeunesses 
coptes marquèrent leur opposition aux gouvernants tout en dénonçant publiquement et de manière virulente l'attitude des représentants de l'Église, jugée trop conciliante à l'égard d'un régime perçu, certes, comme un moindre mal, mais malgré tout complice, actif ou passif, de l'islamisation des espaces collectifs et de l'idiome politique, des exactions anti-coptes et de la diffusion massive d'une littérature hostile au christianisme ${ }^{11}$. À partir de l'année suivante, des manifestants se mobilisèrent dans les rues d'Alexandrie et du Caire, et non plus seulement dans l'enceinte de l'Église. Ils entrèrent en confrontation directe et violente avec les forces de l'ordre à plusieurs reprises, par exemple à la suite de l'attentat commis à l'église des Deux-Saints à Alexandrie, dans la nuit du 31 décembre au 1er janvier 2011, critiquant de ce fait l'attitude gouvernementale aussi bien que l'indéfectible soutien du patriarche au régime ${ }^{12}$. Les groupes formés par de jeunes coptes ou par des prêtres militants, qui se multiplièrent entre 2005 et 2012, rejoignirent, pour certains d'entre eux, le mouvement révolutionnaire ou naquirent pendant le processus insurrectionnel. Le plus souvent, le passage des jeunesses coptes au militantisme s'effectuait dans les réseaux communautaires. Fortes de l'exemple des militants en diaspora et dans d'autres secteurs de la société, motivées par l'expérience révolutionnaire, puis choquées par la répression militaire des manifestants majoritairement coptes sur les quais de Maspéro au mois d'octobre 2011, elles fondèrent plusieurs associations militantes (Coptic Egyptian Federation, Coptic Without Restrictions, Maspero Youth Union...) dans l'objectif de défendre les droits des coptes.

Cependant, la communautarisation sociale se dessina dans la géographie du centre ville cairote. Alors que la place Tahrîr figurait le cœur vibrant de la révolution, de la fierté nationale retrouvée et de l'unité des "deux éléments de la nation ", un autre centre contestataire se forma, en retrait, vers Maspéro, d'où partaient, certes, des manifestations menées par des acteurs sécularistes (tels les membres du groupe MARED ${ }^{13}$, mais surtout les marches de protestation organisées par des associations coptes dont les revendications se concentraient
11. Laure

Guirguis,

"Contestations

coptes

contemporaines ",

in Sarah

Ben Nefissa

et Blandine

Destremau (dir.),

Protestations

sociales,

révolutions civiles.

Transformations

du politique dans

la Méditerranée

arabe, Revue Tiers

Monde, Hors

série 2011, Paris,

Armand Colin,

pp. 139-163 et

Coptes d'Égypte et

Reconfigurations

politiques

2005-2012,

Paris, Karthala

(à paraître,

automne 2012);

Samer Soliman,

"The Radical

Turn of Coptic

Activism: Path

to Democracy

or to Sectarian

Politics", Cairo

Papers in Social

Science, vol. 29,

$n^{\circ} 2 / 3$, Le Caire,

American

University Press,

été-automne

2006, p. 135-155

12. Coptic

Egyptian

Federation,

Coptic Without

Restrictions,

Maspero Youth

Union... 
Fondée en 2005 par l'Anbâ Matiyâs Nasr Manqariyûs, l'association Coptes pour l'Égypte (Aqbât min ajl misr) appela à plusieurs reprises les coptes à manifester dans les rues égyptiennes depuis l'année 2009. Elle organisa une manifestation place Tahrîr après le meurtre de chrétiens à Nig' Hammâdî. Manqariyûs publie depuis 2004 la revue mensuelle Al-Katîba al-tibiyya, « la légion thébaine ", sous supervision officieuse de l'Église, ainsi nommée en référence à une légion de l'armée romaine en Égypte formée de nombreux chrétiens qui devinrent des martyrs pour avoir refusé d'abjurer leur religion, se disant prêts à se battre pour l'empereur mais pas à lui vouer un culte. sur les attaques qui avaient visé les chrétiens et les églises peu après la démission du président. La révolution exacerba les tendances communautariste et nationaliste, relevant toutes deux d'une logique identitaire. La vague insurrectionnelle incita une partie des coptes à sortir de l'espace communautaire pour descendre dans la rue, mais refoula certains d'entre eux derrière les murs de l'Église.

\section{La révolution du 25 janvier et les politiques identitaires nationales et transnationales}

Plus d'un an après le départ du ra'îs, de nombreux coptes, résignés, se retranchent en effet derrière la supposée fatalité d'un destin copte voué au martyre et accordent davantage d'attention à l'élection du successeur de Chenouda qu'aux scrutins nationaux. Au cours des semaines qui suivirent la démission du président, la recrudescence des attaques contre les coptes et l'instrumentalisation intensive des slogans religieux avaient motivé l'expression de revendications axées autour des valeurs communautaires. La fracture communautaire qui gangrène la vie sociale et politique taraude aussi les militances égyptiennes. L'activisme copte, en diaspora et en Égypte, également caractérisé par la diversification des modes d'actions la sectorisation des demandes, la multiplication des acteurs, la communication accrue entre les cercles militants et la prédominance de la référence au droit, oscille entre revendications " citoyennes " et communautaristes.

Cependant, l'oscillation entre ces deux tendances antagonistes se distingue à présent de l'hésitation entre repli sur l'espace communautaire et engagement dans le projet de construction nationale, qui avait de longue date marqué les trajectoires politiques des acteurs coptes. Car l'idéal national ne mobilise plus et n'oriente plus les actions des militants égyptiens, bien que les slogans nationalistes restent prégnants jusque dans l'idiome contestataire (idiome révolutionnaire inclus) et surgissent, véhéments, au moment de dénoncer un 
" ennemi » étranger (fût-il un allié objectif). Certes, le projet national est évoqué, une fois de plus, comme antidote à la «maladie " communautariste; les récits sur l'identité nationale ponctuent les campagnes électorales; la référence à l'unité nationale gouverne le discours médiatique et politicien. Mais cette évocation ne masque pas le souci d'instaurer un État de droit, commun à la plupart des militants politisés qui, certes, ne représentent qu'une infime partie de la société égyptienne.

Sans même évoquer l'indispensable réforme du système éducatif, qui est l'un des véhicules de l'idéologie étatique structurée par une logique identitaire, la résolution de ladite "question confessionnelle " et la levée des discriminations exercées à l'encontre des chrétiens dans la plupart des secteurs de la société égyptienne, suivant des normes énoncées ou des règles tacites, dépendent de trois processus politiques corrélatifs en cours de développement: la reconstruction de l'appareil d'État et, partant, des institutions religieuses; les recompositions du champ islamique égyptien et transnational ; et les dynamiques identitaires mondialisées.

Premier processus, l'articulation entre la restructuration probable de l'institution cléricale et la construction de l'appareil étatique va entraîner une redéfinition des rapports entre l'Église et l'État, entre le patriarche et les gouvernants. Chenouda s'était employé à contrôler tous les rouages de l'institution, tout en favorisant son développement en Égypte et dans les pays d'immigration des chrétiens. Or l'expansion de l'Église copte ne fut pas accompagnée par l'institutionnalisation indispensable à une organisation d'une telle stature. Tout demeurait sous la supervision d'un seul homme dont la volonté avait force de loi. Lors de la nécessaire refonte de l'institution cléricale, des impératifs administratifs et organisationnels dicteront par conséquent au successeur de Chernouda III d'institutionnaliser la décentralisation en cours. Il devra redéfinir les charges et les responsabilités du personnel clérical, diviser et répartir les fonctions que Chenouda s'étaient attribuées. Ces changements au sein de l'Église pourraient favoriser l'expression de la pluralité des orientations politiques des coptes qui se manifesta au cours des dernières années, pour autant que les développe-
Cette revue est diffusée dans les espaces communautaires uniquement, à l'instar de la plupart des publications éditées "par" et " pour " les coptes, sauf l'hebdomadaire Watanî, dont le rédacteur en chef est Yûsif Sidhum, qui est distribué en kiosque. 13. Cf. note 6 . 
ments sur la scène politique nationale le permettent également. Or il est naturellement trop tôt pour savoir comment s'agenceront à l'avenir les relations entre les gouvernants et l'Église. Néanmoins, le sort des chrétiens en Égypte apparaît tributaire de la construction d'un État de droit, doté d'institutions indépendantes du régime et des gouvernants, et qui ne véhicule pas une idéologie identitaire comme c'est le cas de l'État instauré par le régime de juillet.

Deuxième mécanisme: depuis une quarantaine d'années, la variante islamique du thème identitaire a remporté la bataille. Aussi les recompositions politiques et doctrinales au sein de l'islam joueront-elles un rôle crucial dans les évolutions des conceptions de l'autre et de son statut, de son intégration dans la vie politique et sociale égyptienne. L'entrée des courants de l'islam politique dans la compétition électorale, et tout d'abord celle des Frères en 1984, conjuguée à l'exigence de conformer les discours politiques aux normes prônées par les instances internationales, avaient renforcé l'instrumentalisation de la "question copte " à chaque scrutin, de la part de tous les acteurs politiques, islamistes ou pas. Modifiant les modes de politisation d'une multiplicité d'acteurs, la révolution du 25 janvier a déclenché une véritable explosion de la scène politique islamiste. Elle a exposé les dissensions internes à chacun de ces mouvements, en particulier les divergences d'ordre générationnel, en même temps que la pluralité des orientations politiques et religieuses qui les composent.

La position adoptée à l'égard des coptes joue encore le rôle de question-test quant à la capacité des acteurs politiques à manier la rhétorique démocratique ou, plus modestement, celle de l'unité nationale, et de critère distinctif entre les divers courants salafistes aussi bien qu'entre ceux-ci et les autres formations islamistes. Les Frères ont continué à multiplier les signes d'amitié à l'égard des chrétiens, à les inviter à adhérer au Parti Liberté et Justice et à proposer d'engager le dialogue, notamment avec la jeunesse copte et des membres du clergé. Pour sa part, Abû al-Futûh activait ses réseaux professionnels dans les milieux médicaux pour tester son potentiel de sympathie auprès des nombreux chrétiens exerçant une 
profession libérale dans la capitale égyptienne. Parallèlement, les Frères ont réitéré leur position sur l'accès des coptes et des femmes à la présidence de la République, transformé le référendum organisé au mois de mars 2011 en vote pour ou contre l'islam et scandé des slogans hostiles aux chrétiens, lors des élections législatives de 2012, dans les circonscriptions où cette tactique paraissait appropriée. Dans les rangs salafistes, les militants ont déployé des tactiques similaires, la base faisant preuve d'une agressivité que les leaders ne contrôlaient pas toujours. Ainsi, après divers épisodes de violences menées contre les coptes au milieu de l'hiver 2011 à l'instigation d'individus frayant dans la nébuleuse salafiste, le chaykh salafiste Mohammad Hassan a participé à une séance de réconciliation. Les jihadistes repentis, enfin, ont essayé de se plier à ces règles rhétoriques. Dans une formulation sibylline, ils ont tenté de concilier le respect des règles jugées indiscutables de la tradition islamique et, partant, le maintien de la jizya et du quasi-statut de dhimmî, et l'inscription de leur discours dans la logique normative prônée par les instances internationales et, par suite, l'abandon de ce statut en vertu des impératifs dictés par les transformations sociales et le bien commun.

Pour autant, la révolution a entériné l'existence de nouveaux critères de distinction, en deçà de celle existant entre " sécularistes " et " islamistes ": entre les forces conservatrices, représentées par les militaires et les Frères occupant des fonctions de décision, et les forces révolutionnaires; entre les révolutionnaires-réformistes et les révolutionnaires-anarchistes ou autres groupuscules " ultras ", réfractaires à toute forme d'association politique, voire à l'État lui-même, dont les protagonistes sont issus des mouvances de l'extrême gauche aussi bien que des rangs salafistes - salafistes d'abord opposés au mouvement révolutionnaire; entre la ville et la province rurale. Certes, l'élection présidentielle de 2012 confirma la centralité de la question du rôle de l'islam dans les débats égyptiens. Leur virulence s'accrut d'autant plus que la situation tactique s'y prêtait tout particulièrement, Abû al-Futûh pouvant être taxé de libéral ou d'extrémiste, en fonction des interlocuteurs, de la période de son existence à laquelle ces derniers se réfé- 
raient et des impératifs dictés par les stratégies discursives du moment. Mais la victoire électorale remportée par les partis islamistes aux élections législatives marque sans doute la restriction du champ d'application des règles dites islamiques aux mœurs et aux domaines privés tandis que, comme tous les gouvernants contemporains, ils se plient, dans celui de la politique régionale, transnationale et économique, aux impératifs du réalisme politique. Ils représentent par conséquent des interlocuteurs honorables au regard de ceux qui ne souffriront pas quotidiennement des modes contemporains de communautarisation qui, depuis trois décennies en Égypte, tracent dans les corps et les espaces partagés des frontières de plus en plus nombreuses.

Cependant, nul ne peut prédire dans quelles directions s'orienteront, à plus long terme, les jeunesses égyptiennes engagées dans des formations islamistes ou dans des groupes réunissant sécularistes, salafistes et jeunes Frères. Car le fait marquant du mouvement révolutionnaire égyptien réside dans l'émergence, lente, confuse, inorganisée encore, des jeunes générations urbaines. Les dissensions internes qui travaillent la Confrérie des Frères musulmans, par exemple, étaient apparues dès l'année 2005. Pour la première fois, de jeunes militants avaient publiquement critiqué la direction, dénoncé l'organisation fortement hiérarchisée basée sur l'obéissance au chef et exprimé leur désaccord avec la position

14. Patrick

Haenni, « La blogosphère en Égypte. Miroir d'un nouveau militantisme et facteur de tensions internes au sein des Frères musulmans ", Institut Religioscope. Études et analyses, $\mathrm{n}^{\circ} 17$, septembre 2008. de la Confrérie sur les coptes et les femmes ${ }^{14}$. La direction les avait promptement remis au pas et les Frères avaient resserré les rangs, apparemment. Après les journées insurrectionnelles de l'hiver 2011, les jeunes Frères prétendirent former un parti, voire plusieurs. Immédiatement, le guide suprême, Muhammad Badî‘, s'opposa à cette initiative. Malgré cela, plusieurs d'entre eux rejoignirent le Parti du Courant égyptien (Hizb al-tayâr al-masrî), fondé par des militants du Mouvement du 6 avril, par d'autres jeunes activistes et par des Frères musulmans de la jeune génération - tels Muhammad Al Qasas et Ahmad 'Abd al-Gawad, expulsés de la Confrérie à la suite de leur ralliement à une autre formation politique que le Parti Liberté et Justice. Ils s'allièrent à la coalition La 
Révolution continue (Al-thawra mustammara) qui, établie en vue des élections législatives, rassembla plusieurs partis fondés par de jeunes militants peu après la démission de Hosni Moubarak. Pour l'heure, ces nouvelles formations n'ont pas élaboré de discours cohérent ni, par suite, de stratégies de mobilisation. Au contraire, les divers courants salafistes et les Frères disposent d'immenses ressources idéologiques, organisationnelles et financières, et inscrivent leurs actions dans des réseaux de pouvoir et des dynamiques discursives transnationales déterminées.

Enfin, la centralité de l'islam dans les débats égyptiens, sa valeur normative, ne relève plus simplement en effet des dynamiques politiques locales, à savoir le regain des courants de l'islam politique depuis les années 1970 et leur intégration subséquente à la vie politique. Elle ne s'explique pas non plus uniquement en référence à une histoire spécifique, l'histoire d'une décolonisation dont les derniers soubresauts se produiraient de manière d'autant plus violente que les rapports de force contemporains sur la scène politique internationale n'auraient de cesse de réactiver l'image de la "domination coloniale " ou de ses avatars. Si ces facteurs demeurent des clés d'interprétation essentielles, il importe encore de les articuler à des transformations du politique repérables à l'échelle internationale et, en particulier, à deux d'entre elles: la focalisation des débats publics nationaux sur les questions de normes sociales et la prégnance de la notion d'identité dans les discours populistes, souvent fascisants. La situation égyptienne, en dépit de ses spécificités indéniables, s’inscrit par conséquent aussi dans des mutations ressortissant de la professionnalisation de la politique (au sens de policy) et de la dépendance accrue des États aux diktats de l'économie spéculative mondialisée. Ces deux facteurs conjugués ont drastiquement réduit la marge d'action et le pouvoir de décision des acteurs politiques nationaux. Cette évolution a contribué à déplacer les débats sur des questions de normes sociales et d'éthique, parfois essentielles, parfois anecdotiques - bien que l'anecdotique signale en général l'existence d'un problème sociétal fondamental. Elle ne fut pas sans stimuler 
l'élaboration des idéologies identitaires contemporaines en partie produites en réaction aux processus de transnationalisation perçus comme solidaires de l'empiètement sur les souverainetés nationales.

Les mutations du communautarisme égyptien, et les reconfigurations des rapports entre pratiques religieuses, appartenance communautaire et idéal national, apparaissent par conséquent d'autant plus complexes que la longue agonie du nationalisme égyptien, tel qu'incarné par l'État-nation sécuritaire hérité du régime de juillet, se produit aussi en interaction avec ces dynamiques transnationales. Aussi, la sortie de l'idéologie nationaliste et le déclin de l'idéal national, qui pourraient ouvrir la voie à la rupture de la logique identitaire, semblent pour l'heure ne pas interdire sa reconduction selon des modalités inédites et, en particulier, renforcer les communautarismes transnationaux. Rien n'interdit d'espérer que, en Égypte et ailleurs, des forces sécularistes inventent enfin des politiques qui mobilisent l'affect en deçà des dynamiques d'exclusion, qui en appellent au désir, politique, sans impliquer la dénonciation d'un ennemi.

L. G. 


\section{Les Cahiers de l'Orient}

\section{Références}

- GUIRGUIS Laure, "Contestations coptes contemporaines ", in Sarah Ben Nefissa et Blandine Destremau (dir.), Protestations sociales, révolutions civiles. Transformations du politique dans la Méditerranée arabe, Revue Tiers Monde, Hors série 2011, Paris, Armand Colin, pp. 139-163.

- HAENNI Patrick, « La blogosphère en Égypte. Miroir d'un nouveau militantisme et facteur de tensions internes au sein des Frères musulmans ", Institut Religioscope, Études et analyses, $\mathrm{n}^{\circ} 17$, septembre 2008.

- LABÎB Hânî, Al-kanîsa al-misriyya. Tawâzunât al-dîn wa al-dawla [L'Église égyptienne. L'équilibre entre la religion et l'État], Le Caire, Dâr Nahda Misr, 2012.

- ROUSSILLON Alain, "Visibilité nouvelle de la "question copte": entre refus de la sédition et revendication citoyenne", in Florian Kosthall (dir.), L'Égypte dans l'année 2005, Le Caire, CEDEJ, 2006, p. 137-175.

- SOLIMAN Samer, "The Radical Turn of Coptic Activism: Path to Democracy or to Sectarian Politics", Cairo Papers in Social Science, vol. 29, n² 2/3, Le Caire, American University Press, été-automne 2006, p. 135-155.

- ZÂKHIR Kamâl, Al-ilmâniyûn wa al-kanîssa. Sirââat wa tahâlufât [Les laïcs et l'Église. Antagonismes et alliances], Le Caire, s.e., 2009. 